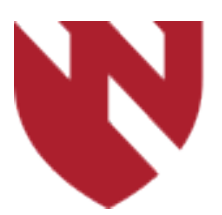

October 2021

\title{
Neuropsychological Predictors of Surgical Outcomes in Medically Refractory Epilepsy
}

\author{
Erin Dennis \\ University of Nebraska Medical Center \\ William Garvey \\ University of Nebraska Medical Center \\ Kaeli K. Samson \\ University of Nebraska Medical Center \\ Matthew Garlinghouse \\ University of Nebraska Medical Center \\ Proleta Datta \\ University of Nebraska Medical Center
}

Tell us how you used this information in this short survey.

Follow this and additional works at: https://digitalcommons.unmc.edu/gmerj

Part of the Higher Education Commons, and the Medicine and Health Sciences Commons

\section{Recommended Citation}

Dennis, E., Garvey, W., Samson, K. K., Garlinghouse, M., , Datta, P. Neuropsychological Predictors of Surgical Outcomes in Medically Refractory Epilepsy. Graduate Medical Education Research Journal. 2021 Oct $04 ; 3(1)$.

https://digitalcommons.unmc.edu/gmerj/vol3/iss1/28

This Conference Proceeding is brought to you for free and open access by DigitalCommons@UNMC. It has been accepted for inclusion in Graduate Medical Education Research Journal by an authorized editor of DigitalCommons@UNMC.For more information, please contact digitalcommons@unmc.edu. 
Neuropsychological Predictors of Surgical Outcomes in Medically Refractory Epilepsy

\section{Creative Commons License}

\section{(c) (1) $\Theta$}

This work is licensed under a Creative Commons Attribution-Noncommercial-No Derivative Works 4.0 License. 
Results: A total of 22,794 charts were queried resulting in 2,513 prescriptions before and 2,648 prescriptions after the inclusion of the calculator. The number of high-risk opiate prescriptions fell by $42 \%(\mathrm{p}<0.001)$, and the average number of MMEs per day decreased from 32.5 , to 31.4 after the intervention $(\mathrm{p}=$ $0.01)$.
Conclusion: Our data show that a likely barrier to complying with the CDC's guidelines regarding safe opiate prescription practices may familiarity with converting narcotic prescriptions to MME, and that embedding a conversion calculator is effective in assisting safe prescription habits.

\section{Neuropsychological Predictors of Surgical Outcomes in Medically Refractory Epilepsy Erin Dennis ${ }^{1}$, William Garvey ${ }^{1}$, Kaeli Samson², Matthew Garlinghouse ${ }^{1}$, Proleta Datta ${ }^{1}$ ${ }^{1}$ University of Nebraska Medical Center, College of Medicine, Department of Neurological Sciences ${ }^{2}$ University of Nebraska Medical Center, College of Public Health, Department of Biostatistics}

Mentor: Proleta Datta

Program: Neurology

Type: Original Research

Background: Resective surgery may be an option for some patients with medically refractory epilepsy. Pre-surgical evaluations including electrophysiology, imaging and neuropsychological tests identify epileptogenic foci and limit risk of postoperative cognitive changes. Our aim is to determine neuropsychological predictors of surgical outcomes to aid in clinical decision making and allow for individually tailored pre-surgical counseling.

Methods: This is a retrospective study of 47 patients who had resective surgery and were followed for 2 years post-operatively. Exclusion criteria included prior epilepsy surgery, lack of neuropsychologic assessment, and neuromodulation device placement or repeat surgery within the follow-up period. Outcome was based on degree of seizure freedom by Engel score with good = I or poor $>/=$ II. EEG, MEG, MRI, or intracranial study abnormalities bilateral or contralateral to the surgical resection were discordant. Non-lateralizing neuropsychologic profiles were not considered to be discordant. Hemispheric dominance was determined by neuropsychologic profile.

Results: A larger proportion of patients had poor outcomes if they had dominant hemisphere resection $(p=0.26)$ or discordant pre-surgical data $(p=0.29)$. There were varying outcomes associated with Wada and specific neuropsychological testing subsets. Median IQ was higher in patients with good outcomes $(\mathrm{p}=0.47)$.
Conclusions: There were no statistically significant associations to suggest that pre-surgical neuropsychological testing can predict post-operative seizure freedom. Limitations include small sample size and that post-surgical neuropsychologic function and quality of life indices were not included. Additional research is needed with larger sample size to further explore the above associations and determine whether these neuropsychological parameters may predict outcomes in other domains.

https://doi.org/10.32873/unmc.dc.gmerj.3.1.015

\section{A Holistic Systems Approach to Characterize the Impact of Chronic Midazolam Exposure on Neurodevelopment and Behavior \\ Mackenzie S. Laurila ${ }^{1,2}$, Nghi M. Nguyen ${ }^{1}$, Kunal Sualy ${ }^{1,2}$, Gurudutt Pendyala ${ }^{1,3}$}

${ }^{1}$ University of Nebraska Medical Center, College of Medicine, Department of Anesthesiology

${ }^{2}$ Children's Hospital and Medical Center, College of Medicine, Department of Anesthesiology

${ }^{3}$ Child Health Research Institute

Mentor: Kunal Sualy, Gurudutt Pendyala

Program: Pediatric Anesthesiology

Type: Original Report

Background: Recent trends in pediatrics are showing increased prevalence of preterm births, as well as significant improvements in neonatal intensive care unit (NICU) mortality. Many of these patients suffer from long term impairments in physical, cognitive, and psychological functions. Midazolam is a short acting benzodiazepine used frequently in the NICU that binds the GABAA receptor causing sedation, anxiolysis, and amnesia. Preclinical studies have correlated early exposure to benzodiazepines with neuroapoptosis and suppressed neurogenesis, however, there are also retrospective studies suggesting pain and stress during the newborn period negatively affect long term neurodevelopment. We hypothesize that chronic midazolam use during early stages of life negatively impacts the developmental trajectory and such changes could extend into adulthood. Using a preclinical rodent model, we are performing a comprehensive characterization of changes in overall physical growth and behavior during adolescence and adulthood.

Methods: Pups were injected with subcutaneous midazolam at escalating doses similar to those seen in the NICU daily from P3 through P21. The control group received isovolumetric saline. Body weight, length, and head circumference were measured. The pups underwent behavioral testing comprised of marble burying and hot/cold plate (nociception) assays. For future molecular studies, brains and blood plasma were isolated from the two groups.

Results: We found a significant reduction in body weight and head circumference in the midazolam group. The marble burying assay demonstrated significantly more obsessive compulsive and anxious behaviors in the midazolam group. Hot and cold plate assays did not show any significant differences in nociception. 
Conclusions: Our findings suggest

phenotypic and behavioral alterations

associated with chronic midazolam exposure, namely reduced body weight and head circumference, as well as increased obsessive compulsive and anxious behaviors in the midazolam group. Future directions for our ongoing study are to assess molecular and biochemical effects on synaptogenesis.

https://doi.org/10.32873/unmc.dc.gmerj.3.1.012

\section{Grant A. Turner ${ }^{1}$, Julie Fedderson², Bethany Lowndes ${ }^{3}$ \\ ${ }^{1}$ University of Nebraska Medical Center, College of Medicine, Department of Internal Medicine, Division of Pulmonology \\ ${ }^{2}$ University of Nebraska Medical Center, College of Medicine, Department of Internal Medicine \\ ${ }^{3}$ University of Nebraska Medical Center, College of Medicine, Department of Neurological Sciences}

Patient Safety Ward of Woe: Using Gamification to Reinforce Patient Safety at a Large Academic Hospital

Mentor: Julie Fedderson

Program: Internal Medicine

Type: Original Research

Background: Healthcare organizations have many priorities, including the education and reinforcement of patient safety and The Joint Commission National Patient Safety Goals (NPSGs). Patient safety escape rooms add gamification to educational programs, adding relevant skills in an enjoyable format. At our institution, all employees undergo patient safety training. We created the "Ward of Woe" to reinforce this training by incorporating errors that were clinical and non-clinical, common and uncommon, and related explicitly to NPSGs.
Methods: In March 2020, an escape room was created using already present materials. All employees were invited to the event. Before entering, the participant was given an SBAR containing information on the simulation. The room contained over 100 errors; each participant was asked to document five. Afterward, the participant was given a small prize. A survey to gather opinions was sent one week later.

Results: 147 participants participated in the event. $87 \%$ of participants felt the training was relevant to their job and $82 \%$ felt it helped them learn about patient safety; $38 \%$ thought it changed their opinion on patient safety. The percentage of clinical or nonclinical errors did not differ between types of participants.
Conclusions: We were able to take previously described patient safety escape rooms, incorporate NPSGs from The Joint Commission, and create a system-wide educational event. This inexpensive event was felt relevant and educational by participants and easily replicated and scaled to other healthcare organizations. Future interventions can work on making virtual offerings and determining the long-term effects of these interventions.

https://doi.org/10.32873/unmc.dc.gmerj.3.1.014

\section{Resident Perception of Respiratory Therapist Directed Asthma Protocol Weaning and the Impact on Education Simone Warrack¹, Nathaniel Goodrich¹, Lauren Maskin¹, Allison Ashford1, Kristy Carlson² ${ }^{1}$ University of Nebraska Medical Center, College of Medicine, Department of Pediatrics 'University of Nebraska Medical Center, College of Medicine, Department of Otolaryngology}

Mentor: Nathaniel Goodrich

Program: Pediatrics

Type: Original Report

Background: Children's Hospital and Medical Center has been utilizing an asthma exacerbation pathway in the inpatient setting for several years. In October 2019 respiratory therapist (RT)-led albuterol weaning was implemented. Though several studies have been published on RT-led weaning of albuterol treatments, the key metrics reported thus far have included cost, length of stay, imaging, and use of steroids. Other studies have analyzed the implementation of pathways but not RT-led weaning protocols on resident education. To our knowledge, our study is to the first to attempt to examine the impact of an RT-led weaning protocol on resident education.

Methods: Pediatric and Medicine-pediatric residents completed electronic anonymous surveys with subjective and objective questions to assess resident comfort and knowledge. Follow-up focus groups were conducted to allow for open dialogue regarding the RT-led weaning protocol.

Results: Fifty seven percent of residents responded to the survey. Most residents felt comfortable assessing and weaning asthmatics. The objective questions revealed that $57 \%$ of residents correctly calculated respiratory scores and a majority of residents could pinpoint when to wean or escalate treatment. Notably, $11 \%$ of residents participated in the focus groups.

Conclusions: Most residents felt comfortable caring for asthmatics and agreed that pathways or protocols support resident education. The residents indicated that hands-on learning was a large component of their education but expressed a need for team approach in asthma care. This study was limited by the fact that few participating residents had actual experience with RT-led albuterol weaning.

https://doi.org/10.32873/unmc.dc.gmerj.3.1.013 